Article

\title{
Fabrication and Evaluation of Multilayer Nanofiber-Hydrogel Meshes with a Controlled Release Property
}

\author{
Rigumula Wu, Rohina A. Niamat, Brett Sansbury and Mandula Borjigin * \\ Department of Chemistry, Delaware State University, 1200 N. Dupont Highway, Dover, DE 19901, \\ USA; E-Mails: rwu14@students.desu.edu (R.W.); rniamat@desu.edu (R.N.); \\ brett.sansbury@wesley.edu (B.S.)
}

* Author to whom correspondence should be addressed; E-Mail: dman@desu.edu;

Tel.: +1-302-857-7181; Fax: +1-302-857-6539.

Academic Editor: Jingwei Xie

Received: 4 June 2015 / Accepted: 14 July 2015 / Published: 17 July 2015

\begin{abstract}
Controlled release drug delivery systems enable the sustained release of bioactive molecules, and increase bioavailability over an extended length of time. Biocompatible and biodegradable materials such as polycaprolactone (PCL) nanofibers and alginate hydrogel play a significant role in designing controlled release systems. Prolonged release of bioactive molecules is observed when these polymer materials are used as matrices independently. However, there has not been a report in the literature that shows how different molecules are released at various rates over time. The goal of this study is to demonstrate a novel drug delivery system that has a property of releasing designated drugs at various rates over a defined length of time. We fabricated multilayer nanofiber-hydrogel meshes using electrospun PCL nanofiber and alginate hydrogel, and evaluated their controlled release properties. The multilayer meshes are composed of sandwiched layers of alternating PCL nanofibers and alginate hydrogel. Adenosine triphosphate (ATP), encapsulated in the designated hydrogel layers, is used as a mock drug for the release study. The exposed top layer of the meshes demonstrates a dramatically higher burst release and shorter release time compared to the deeper layers. Such properties of the different layers within the meshes can be employed to achieve the release of multiple drugs at different rates over a specified length of time.
\end{abstract}

Keywords: electrospun nanofiber; hydrogel; multilayer mesh; controlled release; ATP 


\section{Introduction}

Innovation in nanomaterial and engineering is the driving force of the advancement of regenerative medicine. It is desirable that the scaffolds used in tissue growth possess properties such as biodegradability, cell attachment and growth, and controlled release of bioactive molecules. Biodegradable and biocompatible electrospun nanofibers exhibit structural and topographical features that are conducive for in vitro growth and proliferation of a variety of cells. Another class of scaffolding nanomaterial, hydrogel, not only provides a 3D microenvironment but also is an excellent matrix for the controlled release of bioactive signaling molecules. The combinatorial engineering of nanofibers and hydrogel should generate novel materials with more desirable properties.

Both electrospun nanofibers [1,2] and hydrogel scaffolds [3,4] are used extensively in in vitro for cell growth and tissue engineering. Among various biodegradable and biocompatible polymer materials, polycaprolactone (PCL) is preferentially electrospun to fabricate scaffolds for various applications, ranging from neurite growth [5], cartilage [6,7] and bone engineering [8,9], drug release [10,11], and expansion of genetically modified cancer cells [12,13]. Alginates, natural polysaccharide polymers isolated from a brown seaweed, Phaeophyceae, form hydrogel instantly in the presence of divalent cations (i.e., $\mathrm{Ca}^{2+}$ ), and are used widely as food additives and a drug delivery system [14].

Due to their hydrophilicity and swelling properties, hydrogel is a good medium for the controlled release of hydrophilic drugs $[14,15]$. Release kinetics of a variety of bioactive molecules (including growth factors, proteins, DNA and drugs) from alginate gels have been evaluated in numerous studies [16-18]. Similarly, a hydrophobic polymer PCL is electrospun into nanofiber scaffolds for drug delivery [11,19-21]. While both PCL and alginate hydrogel are widely used in delivery of bioactive molecules, it is difficult to achieve the controlled release (i.e., releasing multiple molecules at different rates over a various length of time) from the monolithic matrices (i.e., PCL or alginate) due to the homogeneity of the encapsulation and diffusion based release mechanisms. Extended or sustained release can be achieved by using blended or monolithic matrix materials; however, the burst release is difficult to circumvent [22]. Extended release of proteins from coaxial PCL nanofibers is demonstrated by using core-sheath nanofibers, in which proteins are encapsulated in aqueous polyethylene glycol (PEG) core and PCL as the sheath of the nanofibers $[19,20]$. The hydrophobic sheath (PCL) prevents the release burst of hydrophilic agents (i.e., proteins) from the core PEG to extend the delivery time. Although the release time can be extended, such a sustained release of bioactive agents from a monolithic scaffold or matrix is not suited to provide multiple bioactive molecules with different concentrations over a various length of time, which is necessary for the different stages of stem cell differentiation [23,24]. Studies of certain growth factors used with controlled release matrices demonstrate that sustained release over a period of two or three weeks enables desired stem cell behaviors (either maintenance of stemness or differentiation) in vitro [25,26].

The aim of this study is to construct multilayer nanofiber-hydrogel meshes with alternating layers of PCL nanofibers and alginate hydrogel for the purpose of releasing multiple bioactive molecules from different layers of a multilayer mesh at different release rates over a various length of time. We fabricated multilayer nanofiber-hydrogel meshes using electrospun PCL nanofibers and $\mathrm{CaCl}_{2}$-triggered polymerization of alginate hydrogel. Adenosine triphosphate (ATP) was used as a drug release model compound, blending in the alginate aqueous solution prior to the gel polymerization. ATP was 
specifically chosen as a mock drug for hydrophilic small molecule growth factors and oligonucleotides. Furthermore, its molecular size is comparable to that of many drugs, vitamins and some hormones. While ATP can be blended into PCL nanofibers, the other hydrophilic bioactive molecules might not be compatible with PCL and the organic solvents. In this study, ATP is readily encapsulated in any specific hydrogel layers of the meshes, and its release in the surrounding can be detected with ultrahigh sensitivity. Commercially available luminescence ATP assay kits detect fM ATP released from a few human cells in a culture medium [27]. To our best knowledge, there is no other release study method reported in the literature as sensitive as ATP assay. Our data show that ATP encapsulated in layers with closer proximity to the aqueous surrounding releases faster than that in the farther distant layers. This finding suggests that such a differential release pattern can be employed to deliver multiple bioactive molecules with different concentrations at different release rates over a various designated length of time. Furthermore, the multilayer construct provides the capabilities of encapsulation of different drugs in different layers and the modulation of their release kinetics individually, and prevents burst release of the drugs.

\section{Experimental Section}

\subsection{Materials}

PCL (MW 80,000 Da), Alginate Sodium (MW 80,000-120,000 Da), and $\mathrm{CaCl}_{2}$ were purchased from Sigma-Aldrich (St Louis, MO, USA) and used as received. Dichloromethane (DCM) and $N, N$-Dimethylformamide (DMF) were purchased from Acros Organics (Monroeville, NJ, USA). Phosphate buffer saline (PBS), penicillin/streptomycin, trypan blue and 24 well plates were purchased from Fisher Scientific (Waltham, MA, USA). The K562 leukemia cell line, Iscove's Modified Dulbecco's Medium (IMDM), and fetal bovine serum (FBS) were obtained from the American Type Culture Collection (ATCC) (Manassas, VA, USA). ATPlite 1 step assay kit was purchased from Perkin Elmer Inc. (Waltham, MA, USA). An ultrasonic cleaner M1800H (Branson Ultrasonics, Danbury, CT, USA) was used to dissolve PCL in solution. In house electrospinning apparatus setup, with components of ES30P-5W high voltage supply (Gamma High Voltage Research Inc. Ormond Beach, FL, USA), syringe pump, syringes, and blunt needles (Thermo Fisher Scientific, Waltham, MA, USA), were used for electrospinning. An oxygen plasma cleaner, PDC-32G (Harrick Plasma, Ithaca, NY, USA), was used to sterilize and make PCL nanofiber mesh surface hydrophilic. A Toggle Press, a $15 \mathrm{~mm}$ circular die, and a Dog-bone shape die were purchased from Pioneer Die-tecs (Weymouth, MA, USA). A Shimadzu Autograph AGS-X with 10 N Load-cell and digital video extensometer (Shimadzu Corp, Columbia, MA, USA) were used to determine Young's Module of the nanofiber meshes. A SpectraMax L luminescence microplate reader (Sunnyvale, CA, USA) was used to perform the ATP release assay. A level A2 biosafety cabinet, an isotemp $\mathrm{CO}_{2}$ incubator and a Life Technologies Countess II automated cell counter were purchased from Thermo Fisher Scientific (Waltham, MA, USA).

\subsection{Electrospun Nanofiber Meshes}

PCL nanofiber meshes were fabricated following the procedure described in Borjigin et al. [12]. Briefly, PCL pellets were dissolved to make a $13.5 \%(\mathrm{w} / \mathrm{v})$ solution in a solvent mixture consisting of 
dichloromethane (DCM) and $N, N$-dimethylformamide (DMF) with a ratio of 4:1 (v/v). An ultrasonic cleaner M1800H (Branson Ultrasonics, Danbury, CT, USA) was used to make homogeneous solutions. The PCL nanofiber meshes were fabricated using an electrospinning apparatus composed of ES30P-5W high voltage supply (Gamma High Voltage Research Inc. Ormond Beach, FL, USA), syringe pump, syringes, and blunt needles (Thermo Fisher Scientific, Waltham, MA, USA). The PCL solutions were pumped at flow rate of $0.5 \mathrm{~mL} / \mathrm{h}$ using a syringe pump while a potential of $12 \mathrm{kV}$ was applied between the spinneret (a 22-gauge needle) and a grounded aluminum foil collector located $12 \mathrm{~cm}$ away from the spinneret.

\subsection{Fabrication of Multilayer Nanofiber-Hydrogel Meshes}

Alginate sodium was dissolved in water to make a $0.5 \%(\mathrm{w} / \mathrm{v})$ aqueous solution. For ATP release assay, a $10 \mu \mathrm{M}$ ATP containing $0.5 \%$ alginate aqueous solution was made by mixing an appropriate amount of stock solution of ATP into the alginate. The ATP encapsulated alginate hydrogel layer was fabricated onto the PCL nanofiber meshes using a two-step procedure: First, $\mathrm{CaCl}_{2}$ solution was applied onto the top surface of the PCL nanofiber mesh by dipping the mesh-attached nanofiber collector briefly into a $0.1 \%(\mathrm{w} / \mathrm{v})$ aqueous $\mathrm{CaCl}_{2}$ solution. Second, alginate hydrogel was facilely fabricated onto the $\mathrm{CaCl}_{2}$ wetted mesh surface by the same fashion of dipping the collector into the $10 \mu \mathrm{M}$ ATP containing $0.5 \%$ alginate solution. The PCL nanofiber layer was electrospun for $20 \mathrm{~min}$ onto the hydrogel surface, and the meshes were treated with an oxygen plasma cleaner PDC-32G (Harrick Plasma, Ithaca, NY, USA) for $5 \mathrm{~min}$ to sterilize and make its surface hydrophilic for the next layer of hydrogel deposition. The cycle of electrospinning, plasma treatment, $\mathrm{CaCl}_{2}$ wetting, hydrogel polymerization was repeated for designated number to make three-, five-, seven-, and eight-layer nanofiber-hydrogel meshes.

\subsection{Scanning Electron Microscopy (SEM)}

Scanning electron microscopy (SEM) was used to visualize the texture of the fiber membranes and the surface topography of the electrospun fibers. The fiber membranes were placed on an aluminum mount-M4 (cat \# 75610) (Electron Microscopy Sciences, Hatfield, PA, USA) and coated with Au/Pt using a Denton Bench Top Turbo III carbon evaporator (Denton Vacuum, Moorestown, NJ, USA) prior to imaging under a Hitachi S4700 Field Emission Scanning Electron Microscope (Hitachi High Technologies, Tokyo, Japan) with magnification between 400 and 6000 folds.

\subsection{Tensile Testing}

Nanofiber meshes along with the aluminum foil collector substrate were cut into a Dog-bone shape using a Toggle Press (Pioneer Die-tecs, Weymouth, MA, USA). The aluminum foil collector at the central region of the dog-bone shape sample was carefully detached from the nanofiber, and was cut to make the specimen alone to be subjected to the tensile force during the measurement. The gauge length and width of the specimen were determined by the Shimadzu Autograph AGS-X micro-tensile tester (Shimadzu Corp, Columbia, MA, USA), and the thickness of the mesh was obtained from the SEM measurement. The tensile testing of the specimen was performed using the Shimadzu Autograph AGS-X micro-tensile tester with a $10 \mathrm{~N}$ load cell and digital video extensometer at ambient temperature and humidity of $65 \%$, setting the cross-head speed at $5 \mathrm{~mm} / \mathrm{min}$. The rigidity (Young's Modulus, 
$E=\frac{F L o}{A o \Delta L}$ ) was calculated using the machine associated software Trapezium $\mathrm{X}$ at the initial linear region of the stress-strain curve.

\subsection{ATP Release Kinetics}

A standard curve of ATP concentration versus luminescence intensity of the luciferin-luciferase reaction was generated using ATPlite one step Assay kit (Perkin Elmer Inc. Waltham, MA, USA) according to the manufacturer's instruction. The dual injection module of SpectraMax L luminescence microplate reader (Sunnyvale, CA, USA) was used to capture the flash luminescence of the reactions. Fifteen millimeter-diameter circular nanofiber-hydrogel meshes along with the aluminum collector were cut using a Toggle Press and a circular die (Pioneer Die-tecs, Weymouth, MA, USA). The triplicate samples of meshes with three-, five-, seven-, and eight-layers were submerged in $0.5 \mathrm{~mL}$ PBS buffer in 24 well plates were subjected to ATP release study. The PBS buffer was collected and replaced with fresh buffer every $24 \mathrm{~h}$, and stored at $-20^{\circ} \mathrm{C}$ until all samples were ready for measurement.

\subsection{Cytotoxicity Assessment}

A leukemia cell line, K562, was used to evaluate the cytotoxicity of the multilayer meshes. The meshes were cut into $15 \mathrm{~mm}$ diameter circular pieces using a circular die and a Toggle Press (Pioneer Die-tecs, Weymouth, MA, USA), sterilized with $70 \%$ alcohol for $30 \mathrm{~min}$, rinsed, and placed in $0.5 \mathrm{~mL}$ Iscove's Modified Dulbecco's Medium (IMDM) (ATCC, Manassas, VA, USA) containing 24 well plates. In each well, $2.5 \times 10^{5}$ cells were seeded, and the medium was changed every other day over the course of an eight-day period. Cell viability was measured every $24 \mathrm{~h}$ using a Countess II automated cell counter (Thermo Fisher Scientific, Waltham, MA, USA).

\subsection{Statistical Analysis}

All treatments and controls for the release study and cell viability assays were conducted in triplicate, and the data are expressed as mean $\pm \mathrm{sd}$. Statistical analysis for release kinetics and cell viability assays were carried out using One Way ANOVA of SigmaPlot 13.0. The difference of the data at $P<0.05$ was considered significant when a more stringent normality test (Shapiro-Wilk) was used. When the difference was not great enough, a less stringent test Kruskal-Wallis One Way ANOVA was conducted at $P<0.05$.

\section{Results and Discussion}

\subsection{Scanning Electron Microscopy Characterization}

The multilayer nanofiber-hydrogel meshes were fabricated using the procedure described in Section 2.3 above, and a diagram is shown in Figure 1A. SEM micrographs show the topography and texture of the different material layers. When an alginate hydrogel is deposited as the top layer, it exhibits a thin film topology. The layer of PCL nanofiber mesh is revealed when a multilayer sample is dried and the top hydrogel layer of the sample is cracked (Figure 1B). The cross section images of the multilayer nanofiber-hydrogel meshes is shown in Figure 1C,D. A freeze-snap treatment of a mesh in liquid 
nitrogen generates an irregular shape cross section edge (Figure 1C), while a neater looking cross section is obtained when the mesh is cut in liquid nitrogen (Figure 1D).

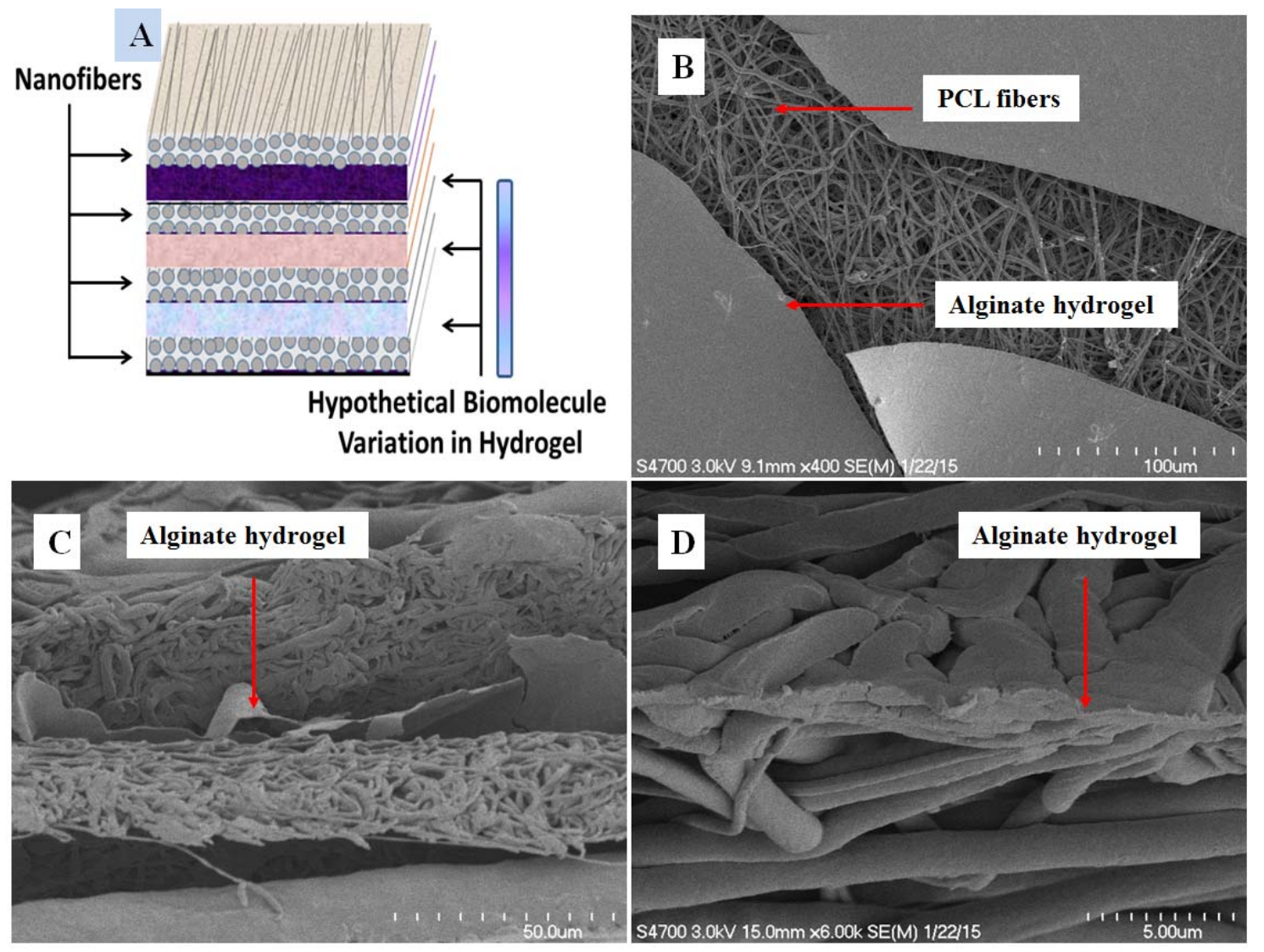

Figure 1. Topographical and texture characterization: (A) Diagram of a multilayer nanofiber-hydrogel mesh, showing the variation of the hypothetical bioactive molecules in the layers; (B) Top view SEM image showing the top two layers of a nanofiber-hydrogel mesh. The top layer alginate hydrogel was cracked to show the PCL nanofiber layer beneath; (C) Cross section SEM image of a multilayer nanofiber-hydrogel mesh, which was snapped in liquid nitrogen; (D) Cross section SEM image of the multilayer nanofiber-hydrogel mesh, which was cut in liquid nitrogen.

During the coating and SEM imaging, the samples were kept dry, and the hydrogel layers appeared as thin films. Therefore, the measurement of hydrogel thickness did not reflect the actual gel thickness in an aqueous environment. Since the PCL nanofiber layers are fairly elastic and malleable compared to the alginate layers in the meshes, it is very difficult to produce a clean-cut cross section. We tried both the freeze-snap method and cutting in liquid nitrogen to generate the cross section of the multilayer samples. The alginate layer tends to shear during the freeze-snap treatment, and cutting in liquid nitrogen generates a smashed cross section.

\subsection{Micro-Tensile Testing}

Rigidity of the meshes was measured under both dry and wet conditions to assess whether the wet environment affects the sample stiffness, which is important for cell growth. Although the PCL nanofiber 
mesh is biodegradable, it does not readily dissolve in water or culture medium. The degradation time in vitro is more than six months, depending on the diameter of the fibers [28]. The rigidity or Young's modulus was determined by calculating the slope of linear region of the stress-strain curve, namely $E=\frac{F L O}{A o \Delta L}$. The rigidity value of the dry nanofiber mesh is $15.59 \mathrm{MPa}$, while that of the wetted PCL nanofiber mesh (soaked in water for $20 \mathrm{~min}$ ) is $11.55 \mathrm{MPa}$ (Figure 2A,B). Apparently, the maximum stress for wetted mesh increases by more than $1.0 \mathrm{MPa}$, its rigidity decreases by $4.04 \mathrm{MPa}$ compared to the dry mesh. It is also observed that the strain (\%) is dramatically increases for the wetted mesh (Figure 2B).
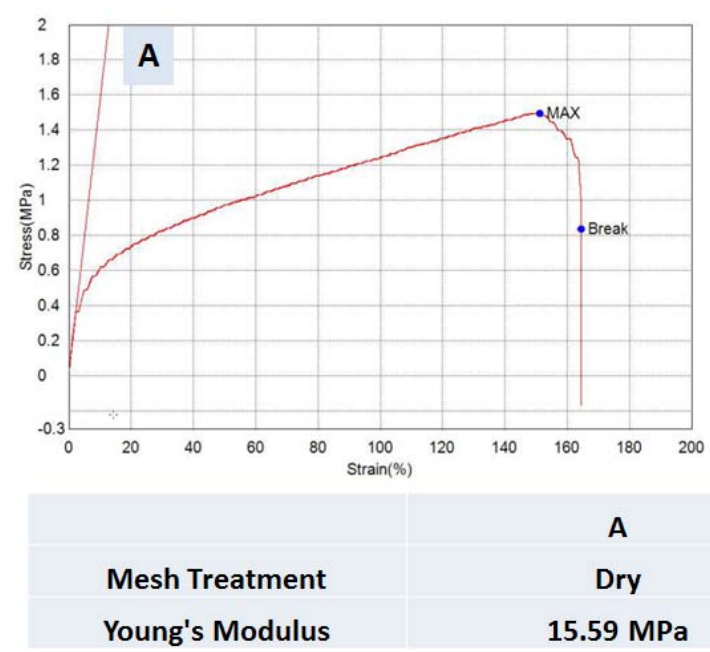

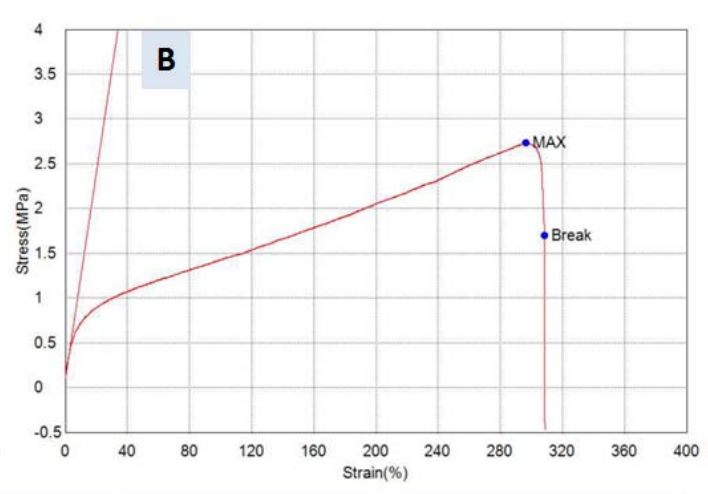

B

Wetted 20 min in water

11.55 MPa

Figure 2. (A) Tensile strength graph of a dry PCL nanofiber mesh; (B) Tensile strength graph of a wet PCL nanofiber mesh, which is soaked in water for $20 \mathrm{~min}$. The elastic modulus values are listed in the table in the figure, showing 15.59 MPa for the dry and $11.55 \mathrm{MPa}$ for the wet samples.

Stiffness of a scaffold is an essential parameter for the biomaterial assessment in tissue engineering and drug delivery $[29,30]$. Optimal stiffness and the strength of a scaffold not only provide a conducive environment for tissue growth but also improve the effectiveness of engraft. Since the intended application of the multilayer nanofiber-hydrogel meshes is in the drug delivery and tissue engineering, the mechanical characterization becomes necessary. The elastic modulus value of PCL nanofiber meshes determined in this study is comparable to those in other studies [31,32], and is even very close to the data generated on single PCL fibers [33].

\subsection{Correlation of Release Kinetics to the Number of Layers}

The commercially available ATP detection assay is extremely sensitive and robust. The correlation between the logarithm of ATP concentration and the logarithm of emitting luminescence intensity possess a linear correlation over a spectrum of seven orders (Figure 3A). It is used as the standard curve to calculate the amount of ATP release throughout this study. The ATP encapsulated meshes were constructed on aluminum foil collector substrates, alternating electrospun nanofiber and hydrogel (Figure 1A). The bottom layer of the multilayer meshes is an electrospun nanofiber layer attached to the aluminum foil collector substrate. The three-layer meshes are consisted of one layer of hydrogel 
sandwiched between top and bottom layers of nanofiber meshes. In the similar fashion, five- and seven-layer meshes have two and three hydrogel layers sandwiched in the nanofiber-hydrogel meshes, respectively. ATP is encapsulated in hydrogel layers by mixing in hydrogel aqueous solution prior to its polymerization on a nanofiber mesh. Bulk release was evaluated with the increasing number of the ATP containing alginate hydrogel layers in the meshes.
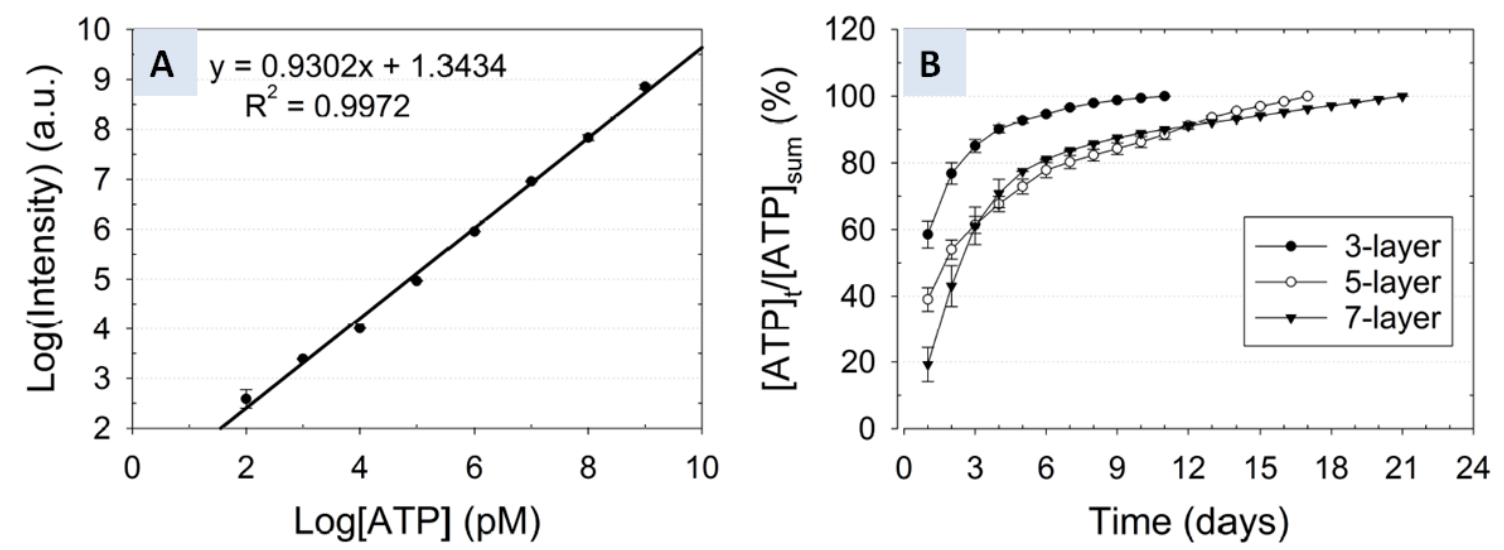

Figure 3. ATP release from cumulative layers of multilayer meshes: (A) Standard curve of ATP concentration vs. luminescence intensity; (B) Cumulative release profile of ATP from a three-layer nanofiber-hydrogel mesh ( $\bullet-)$; Cumulative release profile of ATP from a five-layer nanofiber-hydrogel mesh ( $\_-\_$); Cumulative release profile of ATP from a seven-layer nanofiber-hydrogel mesh ( $\longrightarrow-$ )

The sustained release profile of ATP and the initial release burst pattern for the multilayer are of particular interests to drug release applications. The PCL nanofiber layer is hydrophobic, and plays a role as a barrier between alginate hydrogel and the aqueous environment. Such an insulation or wrapping by PCL layers prevents initial burst release of ATP from the hydrogel. When the insulation layer number increases, the initial release burst should decrease. Similarly, the length of release time can be modulated conveniently by adjusting the layer number of the multilayer nanofiber-hydrogel meshes.

The cumulative ATP release data show that $58.5 \% \pm 4.0 \%$ of the encapsulated ATP is released on day 1 from the three-layer meshes, containing a single hydrogel layer (Figure 3B). In comparison, $39.0 \% \pm 3.5 \%$ and $17.4 \% \pm 5.1 \%$ of initial release burst occurs in the five- and seven-layer meshes, respectively (Figure 3B). The statistical difference between the three-layer mesh data and those of five- and seven-layer meshes is significant $(P<0.001)$ when the data is analyzed with the stringent normality test (Shapiro-Wilk) of SigmaPlot 13.0 One Way ANOVA module. Although the pairwise analysis of all three intact data together does not generate a statistically significant difference, the initial two day data exhibit a significant difference $(P<0.05)$. Following day 3 and on, the normality test on five- and seven-layer samples does not generate a difference at $P<0.01$. This suggests that the difference of the controlled release capacity between five- and seven-layer samples is negligible after a three day release, which might be due to the layer number of the meshes being too few and too close.

Importantly, the trend of the decrease of initial release burst correlates well with the increasing number of the nanofiber layer "insulation or wrapping" between the hydrogel layers. Furthermore, the length of the release time also extends with increasing layer number of the multilayer meshes. While the 
release kinetics of three-layer meshes reaches a plateau before day 11 (Figure 3B), those of five- and seven-layer meshes do not reach until day 17 and 21, respectively. Such a modulation of initial release burst, release at a specific rate and elongation of release time are desired properties of a controlled release system.

\subsection{Differential Release Kinetics for Designated Layers}

Due to the ease of fabrication of the multilayer meshes and drug encapsulation, multiple types of bioactive molecules can be enclosed at various concentrations in different layers of the meshes to achieve the release of a defined amount of different drugs over a specific length of time. To evaluate the capacity of releasing various drugs simultaneously at specific rates but with a different concentration and length of time, we set up a series of simple experiments to study the ATP release kinetics from designated layers of multilayer meshes. Here, ATP is encapsulated in only one designated hydrogel layer of each eight-layer meshes, and the release patterns for different layers are compared (Figure 4). Presumably, the top layer releases ATP faster with a larger burst release, and the buried layers release slower with minimum burst release. Such a differential release kinetics property has advantages of releasing multiple drugs that are discretely encapsulated in designated layers of the multilayer meshes with desired rates and a length of time.

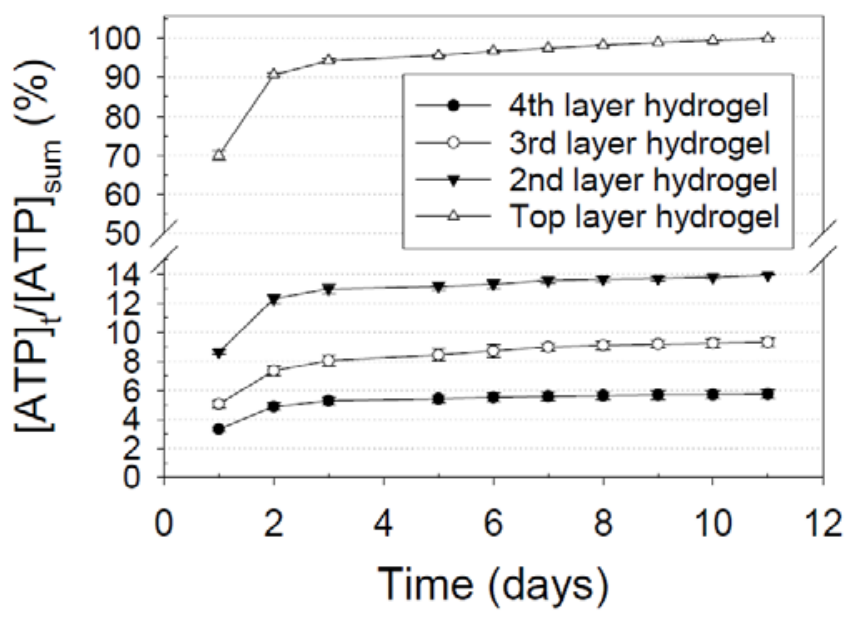

Figure 4. Release kinetics of ATP from a designated layer of the multilayer nanofiber-hydrogel meshes: Release from the top layer hydrogel of an eight-layer nanofiber-hydrogel mesh $(\square-)$; Release from the second hydrogel layer from the top surface of an eight-layer nanofiber-hydrogel mesh ( $\longrightarrow$ ); Release from the third hydrogel layer from the top surface of an eight-layer nanofiber-hydrogel mesh (- - - ) ; Release from the fourth hydrogel layer from the top surface (or the hydrogel layer attached to the bottom nanofiber layer) of an eight-layer nanofiber-hydrogel mesh ( $\left.\bullet-{ }^{-}\right)$.

The release of ATP encapsulated in the top hydrogel layer exhibits remarkable initial release burst characteristics by releasing $69.9 \% \pm 1.2 \%$ on day 1 (Figure 4 ). In contrast, the release burst decreases drastically to $8.6 \% \pm 0.7 \%$ for the 2 nd layer hydrogel ( $3 \mathrm{rd}$ layer from the surface of the eight-layer nanofiber-hydrogel meshes). The release burst for 3rd and 4th hydrogel layers (5th and 7 th layer from 
the surface) is lowered even further to $5.1 \% \pm 0.2 \%$ and $3.3 \% \pm 0.2 \%$, respectively. There is a statistical difference between the data of all layers at $P<0.005$ when the stringent normality test (Shapiro-Wilk) of One Way ANOVA analysis is used. Furthermore, the release amount stays low for the deeper buried hydrogel layers compared to the top layer, suggesting the depletion of the deeper layer encapsulated molecules takes a much longer time. This property can be applied to release different growth factors at different rates and duration of time by encapsulating them in designated layers of the multilayer meshes.

\subsection{Cytotoxicity Assessment of Multilayer Nanofiber-Hydrogel Meshes}

PCL and alginate are FDA-approved biomaterials. Electrospinning process for the fabrication of nanofiber meshes is proven to be safe. Alginate hydrogel is used for many drug delivery systems, and no known adverse effects on in vitro cell culture have been reported. While our long-term intent is to grow and modulate hematopoietic cells, K562 cells are a good model system for such studies. Although collagen blended poly(d,l-lactide-co-glycolide) nanofiber scaffolds used for capturing adherent K562 cells has been reported [34], there is no report on the cytotoxic evaluation of electrospun PCL nanofibers on K562 cells in the literature.

We cultured K562 cells in 24-well plates in the presence of a five-layer nanofiber-hydrogel mesh, and evaluated the cell viability using the LifeTechnologies Countess II automated cell counter over the course of an eight-day period. The viability of the cells onto the mesh is comparable to that on the plate surface, reaching over $97.3 \% \pm 0.6 \%$ (Figure 5). There is no statistical difference between the control and treatment at $P<0.05$ when the stringent normality test (Shapiro-Wilk) of One Way ANOVA analysis is used. When we lower the stringent level and use Kruskal-Wallis One Way ANOVA to analyze the data, there is still no significant difference $(P<0.05)$. These data demonstrate that the multilayer meshes are non-toxic to cells and safe to use for further applications. The viability of the cells for both control and treatment in the initial two days is lower than that in later days, which might be due to the adaptation period of the cell to the new medium and environment.

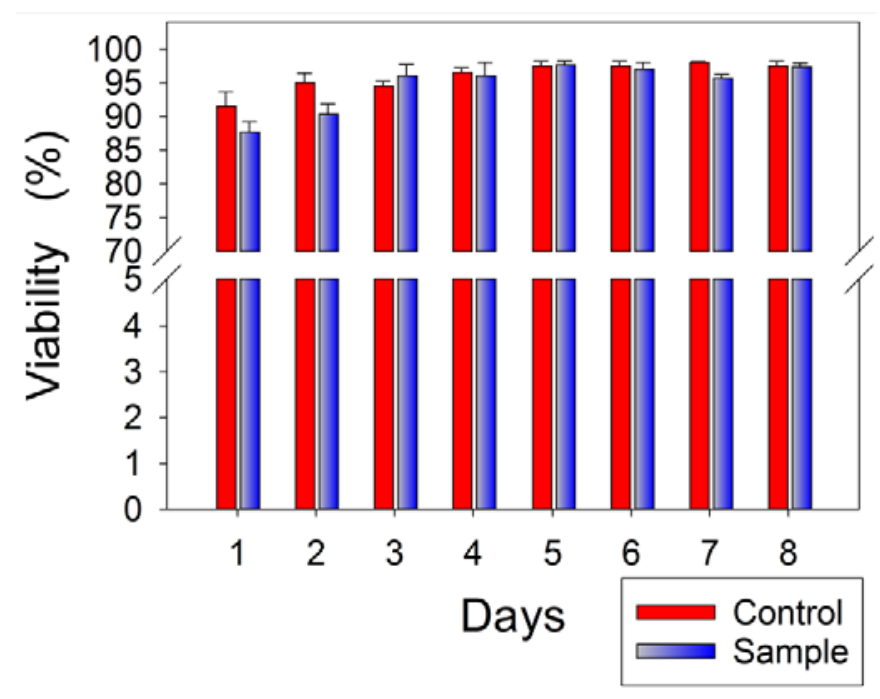

Figure 5. Cytotoxicity assessment of K562 cells on the multilayer nanofiber-hydrogel meshes. The cells grown in regular 24-well plates were setup as the control, and a five-layer specimen mesh as the experimental sample. 


\section{Conclusions}

The results of this study show that the facilely fabricated multilayer nanofiber-hydrogel meshes exhibit genuine controlled release properties, enabling the release of ATP from designated layers with different rates over a specified length of time. ATP encapsulated at the top layer of the meshes is released at a faster rate, a shorter release time, and a dramatically higher release burst compared to that at deeper layers in the meshes. This suggests that the multilayer meshes can be used to achieve the releasing of multiple drugs at different rates over a different length of time by encapsulating them discretely in designated layers.

\section{Acknowledgments}

The authors thank NIH COBRE 1P20GM109021-01. The SEM images were taken with the help of Debbie Powell at the Delaware Biotechnology Institute and Wafa Amir at the Optical Center of Delaware State University. We also thank Eric Kmiec for critical reading and editing of the manuscript.

\section{Author Contributions}

Mandula Borjigin designed experiments, and Rigumula Wu, Rohina Niamat, and Brett Sansbury performed the experiments. Rigumula $\mathrm{Wu}$ and Mandula Borjigin wrote the manuscript.

\section{Conflicts of Interest}

The authors declare no conflict of interest.

\section{References}

1. Pham, Q.P.; Sharma, U.; Mikos, A.G. Electrospinning of polymeric nanofibers for tissue engineering applications: A review. Tissue Eng. 2006, 12, 1197-1211.

2. Vasita, R.; Katti, D.S. Nanofibers and their applications in tissue engineering. Int. J. Nanomedicine 2006, 1, 15-30.

3. Drury, J.L.; Mooney, D.J. Hydrogels for tissue engineering: Scaffold design variables and applications. Synth. Biomim. Polym. 2003, 24, 4337-4351.

4. Park, H.; Karajanagi, S.; Wolak, K.; Aanestad, J.; Daheron, L.; Kobler, J.B.; Lopez-Guerra, G.; Heaton, J.T.; Langer, R.S.; Zeitels, S.M. Three-Dimensional Hydrogel Model Using Adipose-Derived Stem Cells for Vocal Fold Augmentation. Tissue Eng. Part A 2010, 16, 535-543.

5. Xie, J.; MacEwan, M.R.; Li, X.; Sakiyama-Elbert, S.E.; Xia, Y. Neurite Outgrowth on Nanofiber Scaffolds with Different Orders, Structures, and Surface Properties. ACS Nano 2009, 3, 1151-1159.

6. Li, W.-J.; Tuli, R.; Okafor, C.; Derfoul, A.; Danielson, K.G.; Hall, D.J.; Tuan, R.S. A three-dimensional nanofibrous scaffold for cartilage tissue engineering using human mesenchymal stem cells. Biomaterials 2005, 26, 599-609.

7. Casper, M.E.; Fitzsimmons, J.S.; Stone, J.J.; Meza, A.O.; Huang, Y.; Ruesink, T.J.; O’Driscoll, S.W.; Reinholz, G.G. Tissue engineering of cartilage using poly- $\varepsilon$-caprolactone nanofiber scaffolds seeded in vivo with periosteal cells. Osteoarthr. Cartil. 2010, 18, 981-991. 
8. Yoshimoto, H.; Shin, Y.M.; Terai, H.; Vacanti, J.P. A biodegradable nanofiber scaffold by electrospinning and its potential for bone tissue engineering. Biomaterials 2003, 24, 2077-2082.

9. Liu, H.; Ding, X.; Zhou, G.; Li, P.; Wei, X.; Fan, Y. Electrospinning of Nanofibers for Tissue Engineering Applications. J. Nanomater. 2013, 2013, 1-11.

10. Chaturvedi, T.P. Doxycycline Poly E-Caprolactone Nanofibers in Patients with Chronic Periodontitis - A Clinical Evaluation. J. Clin. Diagn. Res. 2013, 7, 2339-2342.

11. Kenawy, E.-R.; Abdel-Hay, F.I.; El-Newehy, M.H.; Wnek, G.E. Processing of polymer nanofibers through electrospinning as drug delivery systems. Mater. Chem. Phys. 2009, 113, 296-302.

12. Borjigin, M.; Strouse, B.; Niamat, R.A.; Bialk, P.; Eskridge, C.; Xie, J.; Kmiec, E.B. Proliferation of Genetically Modified Human Cells on Electrospun Nanofiber Scaffolds. Mol. Ther. Nucleic Acids 2012, 1, e59.

13. Borjigin, M.; Eskridge, C.; Niamat, R.; Strouse, B.; Bialk, P.; Kmiec, E.B. Electrospun fiber membranes enable proliferation of genetically modified cells. Int. J. Nanomedicine 2013, 8, 855-864.

14. Tønnesen, H.H.; Karlsen, J. Alginate in drug delivery systems. Drug Dev. Ind. Pharm. 2002, 28, 621-630.

15. Hoffman, A.S. Hydrogels for biomedical applications. Recent Dev. Hydrogels 2002, 54, 3-12.

16. Jeong, S.I.; Jeon, O.; Krebs, M.D.; Hill, M.C.; Alsberg, E. Biodegradable photo-crosslinked alginate nanofibre scaffolds with tuneable physical properties, cell adhesivity and growth factor release. Eur. Cell. Mater. 2012, 24, 331-343.

17. Gombotz, W.R.; Wee, S.F. Protein release from alginate matrices. Adv. Drug Deliv. Rev. 2012, 64, 194-205.

18. Lee, K.Y.; Mooney, D.J. Alginate: Properties and biomedical applications. Prog. Polym. Sci. 2012, 37, 106-126.

19. Jiang, H.; Hu, Y.; Li, Y.; Zhao, P.; Zhu, K.; Chen, W. A facile technique to prepare biodegradable coaxial electrospun nanofibers for controlled release of bioactive agents. J. Controlled Release 2005, 108, 237-243.

20. Zhang, Y.Z.; Wang, X.; Feng, Y.; Li, J.; Lim, C.T.; Ramakrishna, S. Coaxial Electrospinning of (Fluorescein Isothiocyanate-Conjugated Bovine Serum Albumin)-Encapsulated Poly( $\varepsilon$-caprolactone) Nanofibers for Sustained Release. Biomacromolecules 2006, 7, 1049-1057.

21. Dash, T.K.; Konkimalla, V.B. Poly- $\epsilon$-caprolactone based formulations for drug delivery and tissue engineering: A review. J. Control. Release 2012, 158, 15-33.

22. Huang, X.; Brazel, C.S. On the importance and mechanisms of burst release in matrix-controlled drug delivery systems. J. Control. Release 2001, 73, 121-136.

23. Keller, G. Embryonic stem cell differentiation: Emergence of a new era in biology and medicine. Genes Dev. 2005, 19, 1129-1155.

24. Zhu, Z.; Huangfu, D. Human pluripotent stem cells: An emerging model in developmental biology. Development 2013, 140, 705-717.

25. Lotz, S.; Goderie, S.; Tokas, N.; Hirsch, S.E.; Ahmad, F.; Corneo, B.; Le, S.; Banerjee, A.; Kane, R.S.; Stern, J.H.; Temple, S.; Fasano, C.A. Sustained Levels of FGF2 Maintain Undifferentiated Stem Cell Cultures with Biweekly Feeding. PLOS ONE 2013, 8, e56289. 
26. Zheng, D.; Dan, Y.; Yang, S.; Liu, G.; Shao, Z.; Yang, C.; Xiao, B.; Liu, X.; Wu, S.; Zhang, T.; et al. Controlled chondrogenesis from adipose-derived stem cells by recombinant transforming growth factor-beta3 fusion protein in peptide scaffolds. Acta Biomater. 2015, 11, 191-203.

27. Van Lune, H.; Wiel, J. Method for Detecting ATP. US 6503723 B1, 7 January 2003. Available online: http://www.google.com/patents/US6503723 (accessed on 5 May 2015).

28. Bölgen, N.; Menceloğlu, Y.Z.; Acatay, K.; Vargel, I.; Pişkin, E. In vitro and in vivo degradation of non-woven materials made of poly ( $\varepsilon$-caprolactone) nanofibers prepared by electrospinning under different conditions. J. Biomater. Sci. Polym. Ed. 2005, 16, 1537-1555.

29. Hollister, S.J. Porous scaffold design for tissue engineering. Nat. Mater. 2005, 4, 518-524.

30. Chan, B.P.; Leong, K.W. Scaffolding in tissue engineering: General approaches and tissue-specific considerations. Eur. Spine J. 2008, 17, 467-479.

31. Kim, G.H. Electrospun PCL nanofibers with anisotropic mechanical properties as a biomedical scaffold. Biomed. Mater. 2008, 3, doi:10.1088/1748-6041/3/2/025010.

32. Johnson, J.; Niehaus, A.; Nichols, S.; Lee, D.; Koepsel, J.; Anderson, D.; Lannutti, J. Electrospun PCL in Vitro: A Microstructural Basis for Mechanical Property Changes. J. Biomater. Sci. Polym. Ed. 2009, 20, 467-481.

33. Chan, S.; Yang, H.; Ko, F.; Ayranci, C.; Basu, S. Tensile Stress-Strain Response of Small-diameter Electrospun Fibers. Agil. Technol. 2012, Available online: http://cp.literature.agilent.com/ itweb/pdf/5991-0178EN.pdf (accessed on 28 May 2015).

34. Cao, X.; Kwek, K.; Chan, J.K.Y.; Chan, C.K. H.; Lim, M. Electrospun nanofibers as a bioadhesive platform for capturing adherent leukemia cells. J. Biomed. Mater. Res. A 2014, 102, 523-531.

(C) 2015 by the authors; licensee MDPI, Basel, Switzerland. This article is an open access article distributed under the terms and conditions of the Creative Commons Attribution license (http://creativecommons.org/licenses/by/4.0/). 